\title{
Theoretical Evaluation of the Thermal Conductivity in Framework (Clathrate) Semiconductors
}

Jianjun Dong ${ }^{1}$, Otto F. Sankey ${ }^{1}$, Charles W. Myles ${ }^{2}$, Ganesh K. Ramachandran ${ }^{3}$, Paul F. $\mathrm{McMillan}^{3}$, and Jan Gryko ${ }^{4}$

${ }^{1}$ Department of Physics and Astronomy, Arizona State University, Tempe, AZ 85287,

${ }^{2}$ Department of Physics, Texas Tech University, Lubbock, TX 79409,

${ }^{3}$ Department of Chemistry, Arizona State University, Tempe, AZ 85287,

${ }^{4}$ Department of Physical and Earth Sciences, Jacksonville State University, Jacksonville, AL 36265.

\section{ABSTRACT}

We have calculated the room temperature thermal conductivity in semiconductor germanium clathrates using statistical linear-response theory and an equilibrium molecular dynamics (MD) approach. A key step in our study is to compute a realistic heat-current $J(\mathrm{t})$ and a corresponding auto-correlation function $\langle J(\mathrm{t}) J(0)\rangle$. To ensure convergence of our results and to minimize statistical fluctuations in our calculations, we have constructed large super-cell models (2944 atoms) and have performed several independent long time simulations ( $>1,500 \mathrm{ps}$ in each simulation). Our results show an unexpected "oscillator" character in the heat-current correlation function of the guest-free Ge clathrate frameworks. This is absent in the denser diamond phase and other with simple structural frameworks. We seek to interpret these results using lattice dynamics information. A study of the effects of the so-called "rattling" guest atoms in the openframework clathrate materials is in progress.

\section{INTRODUCTION}

Recent experiments reveal that certain crystalline forms of Ge-based clathrates (e.g. $\mathrm{Sr}_{8} \mathrm{Ga}_{16} \mathrm{Ge}_{30}$ ) have an abnormally low, glass-like thermal conductivity ( $\kappa$ ) [1]. This makes these materials very promising for the design of high ZT thermoelectric materials. However, the mechanism of this reduction of $\kappa$ is still not fully understood. Slack and coworkers have suggested [2] that the small guest atoms (e.g. Sr) located inside the open framework (e.g. clathrate) behave like loosely bound "rattlers", and that their lowfrequency rattling phonon modes scatter the heat-carrying acoustic phonons of the framework. No quantitative theoretical calculation has yet been reported to support this conjecture. We have recently performed a detailed first-principles theoretical study of the lattice dynamics of alloyed Ge clathrates, which showed that interaction between the framework acoustic phonon branches and the Sr-related rattling modes is possible because of avoided crossing [3]. These results provide the lattice dynamics basis to interpret the Slack-proposed rattler scattering mechanism.

It is, however, possible that the large reduction in $\kappa$ is caused by multiple mechanisms. The novel open-framework of the clathrates may also be relevant. In a broader context, the conventional transport formalism in condensed matter physics has been developed based on our understanding of simple crystals, such as diamond. This 
formalism has not been extensively tested with complex materials systems, such as the framework semiconductors.

The unique character of clathrate framework phonon spectra has been discussed in our previous study [4]. Here, we repeat the lattice dynamics study with a simple classical Tersoff potential [5]. The phonon spectra calculated using this approach shown in Fig. 1. We find semi-quantitative agreement between the Tersoff potential results and those of our previous first-principles study [4]. This indicates that the simple Tersoff potential captures the fundamental physics we are interested in here. Since both (guestfree) Ge clathrates and diamond phase Ge contain $100 \%$ four-fold coordinated Ge atoms, the phonon properties of the $\mathrm{Ge}_{46}$ clathrate are comparable with those of diamond phase Ge. The spectrum of the diamond phase (2-atoms per primitive cell) (Fig. 1a) is well known: three acoustic branches in the low and medium frequency region and three optic branches in the high frequency region. If a 64-atom super-cell is used instead, the spectra can be easily interpreted in terms of "Brillouin zone folding" (Fig. 1b). However, the framework clathrate clearly exhibits some new features (Fig. 1c), besides those similar to zone folding effects. The optical branches in $\mathrm{Ge}_{46}$ (Fig. 1c) are "flatter" than the folded acoustic branches in diamond phase Ge (Fig. 1b), which means that they are less effective for heat transport. Some optic modes in $\mathrm{Ge}_{46}$ have relatively larger dispersion, and can be approximately traced as folded acoustic modes. These "acoustic-like" phonon modes are "resonant" with other optic modes. Because of the avoided crossing effect, these branches bend flat at the "crossing points" and some small energy gaps are created. The details about whether and how these unique features in the phonon spectra alter the transport properties are not clear.

In this study, we wish to achieve a better understanding of the heat conduction mechanism in framework clathrate materials and to try to gain insights into the possible "tuning" of the thermal conductivity of such materials in order to optimize their thermoelectric properties. As a first step, we limited our study to Ge diamond and the pure (guest-free) $\mathrm{Ge}_{46}$ clathrate. We find an unexpected "oscillator" feature in the timecorrelation function of the heat current in the $\mathrm{Ge}_{46}$ clathrate framework and we interpret this result in the context of lattice dynamics. The study of effect of guest atoms on thermal conductivity is in progress, and will be reported elsewhere [6].

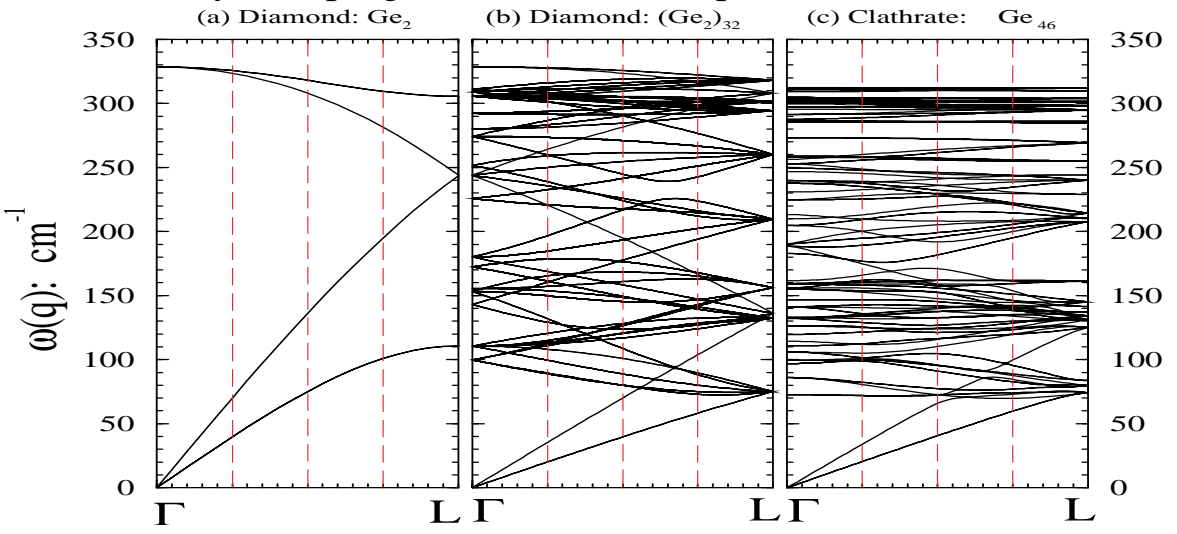

Fig.1 The Tersoff potential calculated phonon dispersion $\omega(\vec{q})$ of (a) Ge diamond in a 2-atom primitive FCC unit cell, (b) Ge diamond in a 64-atom simple cubic super-cell, and (c) $\mathrm{Ge}_{46}$ clathrate (type-I simple cubic framework). $\Gamma$ and $L$ are the labels of $(0,0,0)$ point and $\pi / 2 \mathrm{~L}(1,1,1)$ point respectively, where $\mathrm{L}$ is the lattice size of the cubic unit cell. 


\section{COMPUTATIONAL METHODOLOGIES}

In our current study, the tensor thermal conductivity $(\kappa)$ is calculated based on the Kubo formula of statistical linear-response theory [7],

$$
\kappa_{\alpha \beta}=\frac{V}{K_{B} T^{2}} \int_{0}^{\infty}<J_{\alpha}(t) J_{\beta}(0)>d t, \quad \ldots
$$

where $J_{\alpha}\left(\right.$ or $\left.J_{\beta}\right)$ is the heat current (thermal energy flux) along the $\alpha$ (or $\beta$ ) Cartesian axis at the equilibrium temperature $T$, and $\left\langle J_{\alpha}(\mathrm{t}) J_{\beta}(0)\right\rangle$ is the corresponding time correlation function. $V$ is the volume of the system, and $K_{B}$ is the Boltzmann constant. Since both the diamond and clathrate structures are cubic, $\kappa$ in our study is a scalar and the calculated results are averaged over three Cartesian directions.

We use equilibrium molecular dynamics (MD) simulations to evaluate the heat current function $\vec{J}(\mathrm{t})$, which is [8],

$$
\vec{J}=\frac{1}{V}\left(\sum_{i} \vec{v}_{i} E_{i}+\sum_{i j}\left(\vec{r}_{j}-\vec{r}_{i}\right)\left(\vec{v}_{j} \frac{\partial E_{i}}{\partial r_{j}}\right)\right), \ldots
$$

where $E_{i}$ is the total energy of the $\mathrm{i}^{\text {th }}$ atom, and $\vec{r}_{i}$ and $\vec{v}_{i}$ are the position and velocity vectors of the $i^{\text {th }}$ atom respectively.

The advantage of this MD approach is that anharmonic phonon scattering is naturally built into the interaction potentials, and no further assumption is required. Therefore, it is simple and straightforward. A disadvantage of the method is that it is classical and is expected to be less accurate at low T. Similar implementations of this approach have been adopted to study the thermal conductivity of crystals with point defects [9], amorphous materials [10], and hydrate clathrates [11]. There are several factors that significantly influence the quality of such calculations. (1) The time correlation functions of heat current decay slowly for most crystalline materials, and the integration in Eq. (1) shows poor convergence. We have found that to obtain a good ensemble average, the number of simulation steps must be of the order of a million. (In our studies, a time step of $1 \mathrm{fs}$ is used.) This rules out the possibility of using very accurate yet computationally intensive, first-principles MD method. In this study, we adopt a simple Tersoff potential [5], and believe it is a reasonable approximation for addressing the fundamental physics of the transport properties. (2) A large super-cell model has to be constructed in the MD simulation to have a realistic sampling of the $k$ point phonon modes. A small size model (e.g. a $2 \times 2 \times 2$ super-cell model) may introduce finite-size artifacts.

\section{THERMAL CONDUCTIVITIES: DIAMOND vs. FRAMEWORK CLATHRATE}

We first studied the thermal conductivity of the diamond structure Ge crystal at room temperature $(T=300 \mathrm{~K})$. It can be used to test our numerical implementation by comparison with experiments and to compare with those of the framework semiconductors.

We start with a 512-atom super-cell model of Ge diamond at zero temperature. Then, we raise the temperature to $300 \mathrm{~K}$, and equilibrate the system, first under constant temperature, then under constant energy conditions for about 80,000 time steps at each stage. After this equilibration period, we allow it to evolve (total energy conserved) using 
Newton dynamics for 1,500,000 time steps (1,500 ps). We calculate the heat current vectors at each simulation time step using Eq. (2). The time averaged heat current correlation functions (Eq. (2)) are calculated over a 300 ps range using the 1,500,000 sampled MD simulation results. We find that in each of calculations described above, statistical fluctuations in the correlation function are not negligible for long times $(t>100$ ps) and that multiple calculations starting from different configurations must be averaged to minimize statistical errors. In the study of Ge diamond, nine independent simulations were performed. The averaged normalized correlation function (divided by $\langle\mathrm{J}(0) \mathrm{J}(0)\rangle$ ) is plotted in Fig. 2a.

As expected, the heat current correlation function in Ge diamond decays for long time. It is not totally surprising that this decay in Ge diamond does not strictly follow an exponential law. However, an approximate fitting to a $\beta^{*} \exp (-t / \tau)$ function gives an effective lifetime parameter $\tau \approx 50$ - 60 ps (depending on the choice of fitting range). We also notice that the normalized correlation function rapidly drops from unity to about 0.4 within less than 0.1 ps. This rapid lose of correlation is not fully understood. However, it is seen in other studies such as in crystalline $\mathrm{SiC}$ [9].

Using Eq. (1), we find that thermal conductivity, $\kappa_{\text {diamond, }}$ of Ge diamond at $300 \mathrm{~K}$ is about $114.5 \mathrm{Wm}^{-1} \mathrm{~K}^{-1}$ at $300 \mathrm{~K}$, which is about twice the experimental measured value of $62 \mathrm{Wm}^{-1} \mathrm{~K}^{-1}$ [12]. Nevertheless, the agreement is reasonable since we have not explicitly fit any anharmonicity parameters. Also our calculations assume a perfect single crystal with no defects or grain boundaries. The calculated results reflect an upper-bound for the thermal conductivity.

The thermal conductivity of $\mathrm{Ge}_{46}$ clathrate is calculated using the same numerical techniques. The averaged heat current time correlation function is shown in Fig. 2b. In the present study, a large 2944-atom model (a 4x4x4 super-cell, with a unit cell of 46 atoms) is used in the MD simulations, and the final results are averaged over 3 independent $1,500,000$ step simulations.

We obtain the unexpected results that the correlation function for the Ge clathrate does not follow a simple decay function; rather it decays like a damped oscillator. Even though the envelope of the correlation function in the Ge clathrate is remarkably similar to that of the Ge diamond phase, the cancellation due to these oscillations in the time integration in Eq. (1) significantly reduces the thermal conductivity.

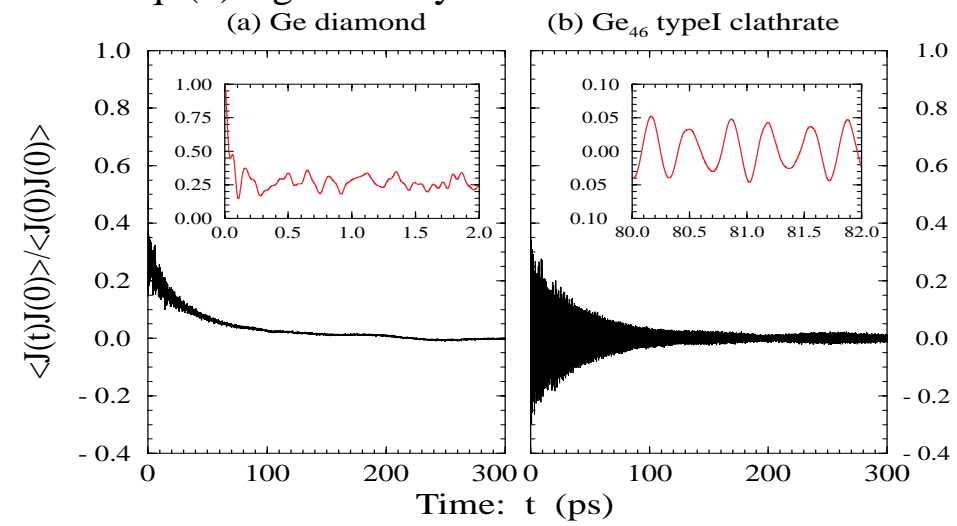

Fig.2 The normalized time auto-correlation functions of (a) Ge diamond, and (b) $\mathrm{Ge}_{46}$ clathrate. The results are calculated using equilibrium MD simulations over a time range of 1,500 ps. 
Therefore, we find a much smaller thermal conductivity in the pure (guest-free) Ge clathrate than in diamond phase Ge. Our theoretical $\kappa_{\text {clathrate }}$ at $300 \mathrm{~K}$ is $12.8 \mathrm{Wm}^{-1} \mathrm{~K}^{-1}$, almost $1 / 9$ of the calculated $\kappa_{\text {diamond }}$ at the same temperature. This is the most significant result of our study.

No pure $\mathrm{Ge}_{46}$ clathrate has yet been synthesized. The most relevant experimental data is the thermal conductivity of metal-doped, alloyed Ge clathrates, such as $\mathrm{Sr}_{8} \mathrm{Ga}_{16} \mathrm{Ge}_{30}, \mathrm{Eu}_{8} \mathrm{Ga}_{16} \mathrm{Ge}_{30}$, and $\mathrm{Sr}_{4} \mathrm{Eu}_{4} \mathrm{Ga}_{16} \mathrm{Ge}_{30}$, for which $\kappa$ ranges from 0.6 to $1.0 \mathrm{Wm}^{-}$ ${ }^{1} \mathrm{~K}^{-1}[1]$. Experiments find that $\kappa$ for compound Ge clathrates containing guests is reduced by a factor of $\approx 60$ compared to diamond Ge. Measurements do not address what proportion of this decrease is due to the guest "rattlers" and what proportion is due to the framework itself. Our calculations suggest that the framework itself (without guest atoms and alloy disorder) reduces the thermal conductivity by a factor of 9 . From this we conclude that the framework and the guest contribute comparable amounts to the reduction of thermal conductivity in the experiments.

In order to better understand the origin of the significant differences in the time correlation functions of the Ge diamond and clathrate structures, we have calculated the (frequency domain) power spectra (Fig.3). We should point out that the "wiggles" at short time in the Ge diamond correlation function (Fig. 2a) are not random noise since they barely change when the function is averaged over 9 independent calculations. These "wiggles" show their signatures in the non-zero frequency part of the power spectra (Fig. 3a). These frequencies are within the phonon frequency region. However, the weight of non-zero frequency components is much smaller than the dominant weight of the zero frequency components.

The most important changes in the power spectra (Fig. 3b) of the $\mathrm{Ge}_{46}$ correlation function are the significant decrease of in the zero frequency peak height and that several relatively high peaks in the finite-frequency region (again, within the phonon frequency range) appear. The large peaks explain the oscillator features in the time correlation function. However, the physical origin of the peaks is not clear. The two dominant peaks are located near $96 \mathrm{~cm}^{-1}$ and $137 \mathrm{~cm}^{-1}$ respectively. On examining the phonon spectrum in detail (Fig. 1c) we have found four different phonon eigen-modes near these two frequencies, which satisfy the energy and momentum conservation conditions for Uprocess phonon scattering. The existence of such four-phonon scattering is plausible. However, we cannot explain why this phonon scattering gives such a large weight in the power spectrum; presumably many other phonon scattering processes are also possible.
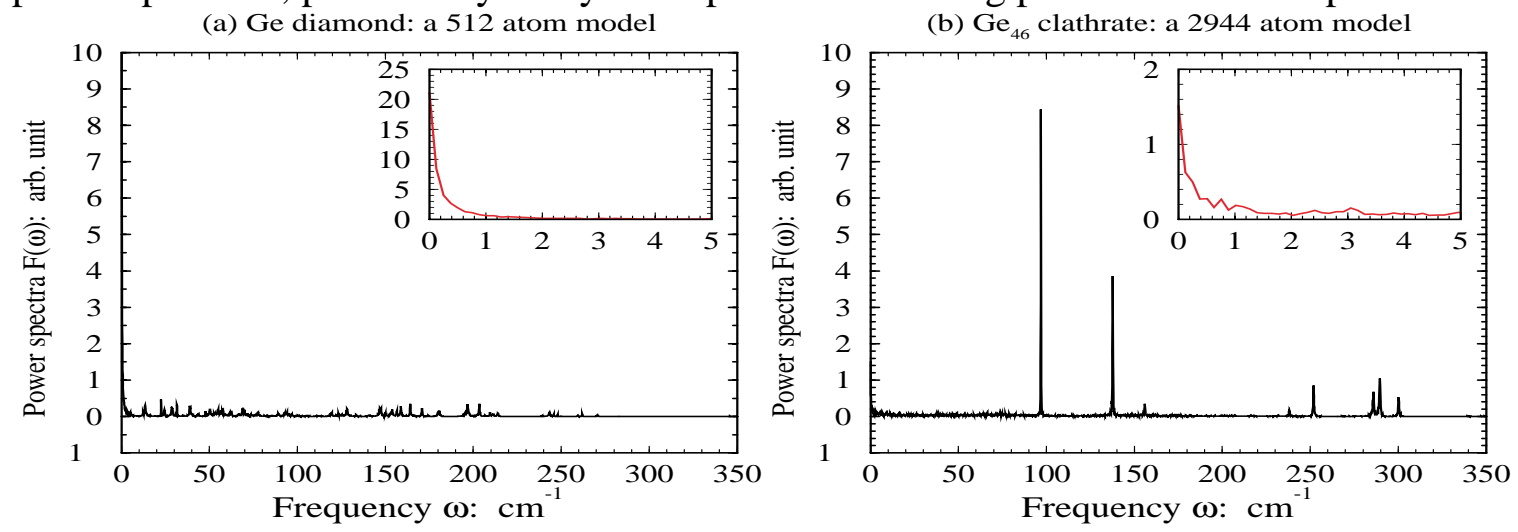

Fig.3 Power spectra of time auto-correlation of (a) Ge diamond, and (b) $\mathrm{Ge}_{46}$ clathrate. 


\section{CONCLUSIONS}

The room temperature thermal conductivity of Ge diamond and $\mathrm{Ge}_{46}$ clathrate materials has been calculated using the Kubo formula and equilibrium MD simulation techniques. Theory shows that the intrinsic thermal conductivity $\kappa$ in the framework clathrate semiconductor is only about $1 / 9$ of $\kappa_{\text {diamond. This reduction in the intrinsic }}$ thermal conductivity $\kappa_{\text {clathrate }}$ can be understood in terms of the rapid oscillations found in the time heat current correlation function. A frequency analysis shows that the oscillation frequencies of the correlation function lie within phonon frequency range. This suggests that heat current in the clathrate strongly couples with several specific phonon modes. The nature of this phenomenon needs further analysis. The effect of guest rattlers has been neglected in this report, and will be reported elsewhere.

\section{ACKNOWLEDGEMENTS}

This work is supported by the NSF-ASU MRSEC (Grant No. DMR-96-32635) and the NSF (Grant No. DMR-99-86706). One of the authors (JD) would like to thank Dr. J.L. Feldman (Naval Research Lab) and Dr. J.S. Tse (NRC, Canada) for insightful discussions. CWM thanks the Department of Physics and Astronomy at Arizona State University for their hospitality while a portion of this work was done.

\section{REFERENCES}

1. G.S. Nolas, J.L. Cohn, G.A. Slack, and S.B. Schujman, App. Phys. Lett. 73, 178180 (1998); J.L. Cohn, G.S. Nolas, V. Fessatidis, T.H. Metcalf, and G.A. Slack, Phys. Rev. Lett. 82, 779-782 (1999).

2. G.A. Slack, in CRC Handbook of Thermoelectrics, ed. M. Rowe (CRC Press, Boca Raton, FL, 1995), pp. 407.

3. J. Dong, O.F. Sankey, G.K. Ramachandran, and P.F. McMillan, J. Appl. Phys, in press (2000).

4. J. Dong and O.F. Sankey, J. Phys.: Cond. Matt. 11, 6129-6145 (1999).

5. J. Tersoff, Phys. Rev. B 39, 5566-5568 (1989).

6. J. Dong and O.F. Sankey, unpublished.

7. R. Kubo, Rep. Prog. Phys. 29, 255 (1966).

8. R.J. Hardy, Phys. Rev. 132, 168-177 (1963).

9. J. Li, L. Porter, and S. Yip, J. Nuc. Mater. 255, 139-152 (1998).

10. Y.H. Lee, R. Biswas, C.M. Soukoulis, C.Z. Wang, C.T. Chan, and K.M. Ho, Phys. Rev. B 43, 6573-6580 (1991); M.D. Kluge, J.L. Feldman, and J.Q.

Broughton, Molecular dynamics simulations of thermal conductivity in insulating glasses, Phonon Scattering in Condensed Matter VII, ed M. Meissner and R.O. Pohl (Springer-Verlag, 1993) pp.225-226.

11. R. Inoue, H. Tanaka, and K. Nakanishi, J. Chem. Phys. 104, 9569-9577 (1996).

12. G.A. Slack and C.J. Glassbrenner, Phys. Rev. 120, 782 (1960). 